\title{
Propuesta de un método de ordenador para resolver el ajuste a la ecuación de Zener-Hollomon (Garofalo) ${ }^{(\bullet)}$
}

\author{
I. Rieiro ${ }^{(*)}$, M. Carsi $^{(*)}$ y F. Peñalba ${ }^{(* *)}$ \\ Resumen Se presenta un método de ordenador integrado, desarrollado por los autores, para resolver la \\ ecuación de Zener-Hollomon. Este método resuelve el problema del ajuste de la ecuación de Zener- \\ Hollomon para amplios rangos de tensión aplicada, velocidad de deformación y temperatura de \\ trabajo, mediante la aplicación progresiva de tres tipos de algoritmos. En primer lugar, una regresión \\ reiterada multivariante por planos, para obtener unos valores que serán considerados como iniciales \\ para un segundo bloque de algoritmos, además de depurar la base experimental de datos. El segundo \\ bloque de algoritmos realiza un ajuste por mínimos cuadrados no lineales usando una modificación \\ del método de Gauss-Newton modificado. En tercer lugar se encadena un grupo de programas que \\ permiten iterar a partir de la solución del programa nó lineal acercándose (si es preciso) por líneas en \\ $R^{2}$ hasta alcanzar la solución óptima. Todo ello con un soporte de análisis estadístico de la bondad de \\ cada paso, de los datos y los residuos y del ajuste final conseguido.
}

Palabras clave: Zener-Hollomon. Garofalo. Métodos numéricos.

\section{Proposal for a computational method to solve the Zener- Hollomon equation (Garofalo)}

\begin{abstract}
An integrated computational method to solve the Zener-Hollomon equation has been developed by the authors. This method solves the fitting problem of the equation of Zener-Hollomon for wide ranges of applied stress, strain rate and work temperature by means of the progressive application of three kinds of algorithms. In the first place a program, by means of a reiterated linear regression by planes, allows to get some values that will be later the initial values of a second block of algorithms. It permits also to purify the experimental data set. The second block of algorithms carries out a fitting by nonlinear least squares using a variation of the modified Gauss-Newton method. In the third place a group of programs is chained that permit iterate starting by the solution of the previous program approaching (if it is necessary) line by line in $R^{2}$ until the optimum value is reached. All this with a support of statistical analysis for they determine the accuracy of each step, of the data, of the residuals and of the last fitting reached.
\end{abstract}

Keywords: Zener-Hollomon. Garofalo. Computer solutions.

\section{INTRODUCCIÓN}

El comportamiento de distintos tipos de aceros, sometidos a ensayos de torsión en condiciones variables de tensión aplicada, temperatura de traba-

(•) Trabajo recibido el día 20 de octubre de 1995

(*) Centro Nacional de Investigaciones Metalúrgicas (CSIC). Avda. de Gregorio del Amo, 8. 28040-Madrid (España).

(**) INASMET. Camino de Portuetxe, 12. 20009-San Sebastián (España). jo y velocidad de deformación, es de gran interés, porque permite determinar sus características mecánicas, tales como resistencia mecánica o ductilidad. También porque se pueden obtener características microestructurales de los materiales, tales como tamaño de grano y de subgrano, fracción volumétrica de material recristalizado y tiempo de recristalización, y de todo ello se pueden realizar predicciones. Las condiciones de tensión y temperatura de trabajo son generalmente las de fluencia por trabajo en caliente con el material policristalino en 
situación de equilibrio entre procesos de restauración y recristalización, para temperaturas homólogas superiores a 0,5. Para la zona estacionaria en velocidad de deformación, se han usado diferentes leyes empíricas que relacionan tensiones, temperaturas y velocidades de deformación, p.e., ley potencial y otras, según el rango de tensiones y del material del que se trate. Pero estas leyes no tienen una validez general en todos los rangos de trabajo. La ecuación de Garofalo o de Zener-Hollomon sí parece dar una respuesta correcta a amplios rangos de $\sigma$ (tensión), $\dot{\epsilon}$ (velocidad de deformación), y $T$ (temperatura). Además, se cree que esta ecuación está soportada por modelos físicos teóricos, los cuales la justifican, que están basados en la estructura del material a nivel de red atómica. Los modelos físicos teóricos son diversos, bien se trate de deslizamiento de fronteras de grano, difusión por vacantes intersticiales, deslizamiento de dislocaciones, trepado de dislocaciones, etc. Este soporte físico teórico para la ecuación de Zener-Hollomon

$$
\dot{\epsilon}=A \cdot e^{\frac{-\mathrm{Q}}{\mathrm{R} \cdot \mathrm{T}}} \cdot[\operatorname{senh}(\alpha \cdot \sigma)]^{\mathrm{n}}
$$

donde

$$
\begin{aligned}
& \dot{\epsilon}=\text { Velocidad de deformación, } \mathrm{s}^{-1} \\
& \sigma=\text { Tensión aplicada, } \mathrm{MPa} \\
& T=\text { Temperatura } \\
& A, Q, \alpha, n=\text { constantes }
\end{aligned}
$$

le permite dotar de significado físico a las constantes que aparecen en la misma y eso hace aún más interesante la obtención de los valores de la ecuación que mejor ajustan la misma a los conjuntos de datos experimentales, y hace necesario que la obtención de estas constantes sea lo más precisa posible. Se cree que en la constante $A$ se pueden incluir factores exponenciales de variación de entropía producida por el tipo de fenómeno que desencadena el deslizamiento, así como parámetros de interés físico, como el vector de Burgers, y según los modelos, frecuencias de vibración propias de la red atómica o de las líneas de dislocación. La constante $Q$ es una energía de activación (entalpía) propia del proceso predominante y se cree que puede incluir valores del campo de tensiones internas generadoras de una activación de carácter no térmico. Se cree que la constante $\alpha$ representa un cierto volumen de activación ponderado por el número medio de elementos que participan en el proceso básico de deslizamiento, y es posible que la constante $n$ esté relacionada con el número medio de elementos constituyentes que participan en el deslizamiento básico. Pero la ecuación de Garofalo es una ecuación compleja desde el punto de vista de la calidad de los valores obtenidos a partir de la misma, para todo el rango posible de valores de interés en tensiones y temperaturas. Es acusada- mente no lineal para dos de los parámetros $(\alpha$ y $n)$ y condicionalmente lineal para otros dos $(Q$ y $A)$. Por lo tanto, el problema matemático se reduce a ajustar la mejor función de la familia de las que tienen la forma [1] a un conjunto de datos obtenidos en ensayos de torsión o de otro tipo $\left\{\dot{\epsilon}_{(\mathrm{i})}, \sigma_{(\mathrm{i})}, \mathrm{T}_{(\mathrm{i})}\right\}$ con $i=1$ ... $n$, obteniendo como resultado $\{A, Q, n, \alpha\}$, valores óptimos del ajuste.

En el análisis de regresión no lineal existen unos tópicos a los cuales se debe ajustar el tratamiento matemático del problema (1-4), que son: a) La función objetivo es correcta. b) La respuesta es la función objetivo más la distorsión. c) La distorsión presenta una distribución normal. d) Cada una de las distorsiones tiene una distribución normal. e) Cada una de las distorsiones tiene una media cero. f) Las distorsiones tienen igual varianza. g) Las distorsiones tienen distribuciones independientes. h) La distorsión proviene de errores experimentales.

Los métodos habituales utilizan la linealización del problema usando un método de Gauss-Newton, con o sin factor de paso, con controles de convergencia, como un relative offset convergence criterion, también son usuales el método directo por suma de cuadrados, el método de Newton-Raphson y las modificaciones de Levenberg-Marquardt al método de pasos descendentes. No obstante, en la literatura se suelen utilizar "métodos iterativos" diversos, no muy especificados. Es conocido que a los criterios más arriba expuestos hay que añadir, en los problemas no lineales, dos condicionantes muy importantes. Por un lado, la gran dependencia para la convergencia y la velocidad de la misma de los valores iniciales, y por otro la posibilidad de obtención de puntos de equilibrio que no sean mínimos en el proceso de optimización de la función de error, bien por ser puntos de silla o resultados espúreos debidos a las características de los datos o de la superficie de ajuste. En el caso que nos interesa, la superficie de ajuste está configurada por zonas abruptas con valores del gradiente muy intensos. $\mathrm{La}$ función a optimizar es:

$\operatorname{SSE}(\theta)=\sum_{i=1}^{i=n}\left(\dot{\boldsymbol{\epsilon}}^{*}{ }_{i}-\theta_{0} \cdot e^{\frac{-\theta_{1}}{x l^{*}}} \cdot \operatorname{senh}\left(\theta_{3} \cdot x 2 *_{\mathrm{i}}\right)^{\theta_{2}}\right)^{2}$

los superíndices estrella indican valores experimentales y el vector $\left\{\theta_{\mathrm{i}}\right\} i=0 \ldots 4$ sustituye a las constantes explicadas. La simple forma de la expresión para un conjunto de datos de varias decenas hace obvia la gran dificultad de la forma de la superficie de ajuste.

\section{CONSTRUCCIÓN DE LOS CÓDIGOS}

En primer lugar, se ha construido un código que linealiza la ecuación de Zener-Hollomon para todas 
las constantes, excepto para una de ellas, $\theta_{3}$. Esta es recorrida en un intervalo definido por los valores físicamente admisibles del problema, con un paso de discretización adecuado, de forma que $\left[\Delta \theta_{3} /\right.$ $\left.\theta_{3 \text { (óptimo) }}\right]<\tau$, siendo $\tau$ determinante de la precisión del valor óptimo. El código realiza múltiples ajustes para los valores sucesivos del discretizado de la constante seleccionada, de todos los ajustes se selecciona el mejor mediante el coeficiente de determinación global corregido $R^{2}$; obtenido el valor óptimo se pasó a una rutina del código en la cual se determina la tabla de ANOVA y, de ella, mediante la obtención de la distribución de la función de distribución experimental $F$ de Snedecor se controla por la validación de hipótesis, la bondad del ajuste, y mediante la validación de las hipótesis correspondientes se determina la bondad de cada una de las constantes obtenidas haciendo uso de la distribución experimental $t$-Student, a partir de ello se obtienen intervalos de confianza para cada uno de los parámetros de la ecuación de Zener-Hollomon obtenidos mediante el ajuste linealizado por planos. Este código, a su vez, entrega unos residuos $e(i)=\dot{\epsilon}(i)^{*}-\dot{\epsilon}(i)$ para $i=1 \ldots n$ a los que somete a un estudio de normalidad, de independencia y de presencia de outliers, obteniendo, además de otros parámetros, una función de frecuencias de los mismos y una función de autocorrelación de los residuos. Este primer código permite caracterizar el problema, respecto de la calidad del conjunto de datos experimentales, de la función de distribución a que responden y de una posible selección y eliminación de puntos que puedan presentar anomalías de base experimental.

El segundo código implementado se basa en unas rutinas que aplican un método no lineal de ajuste por mínimos cuadrados tipo Newton modificado, con factor de paso y control de convergencia que toma como valor inicial el óptimo entregado por el código anterior. Este segundo código también está provisto de rutinas para el análisis exhaustivo de los residuos, que son comparados con los anteriormente obtenidos. Terminado este segundo código, se ha obtenido un conjunto de soluciones con bastantes garantías. Estos resultados se someten al tratamiento de un tercer código que consiste, en esencia, en el trabajo enlazado de dos rutinas, cada una de las cuales permite realizar ajustes sobre el espacio $\mathbb{R}^{4}$, moviéndose en cortes sobre dos dimensiones, o sea en $\mathbb{R}^{2}$; en cada uno de los cortes se ha seleccionado una de las constantes del ajuste de la ecuación de Zener-Hollomon, bien $Q$ o bien $\alpha$, tomando para ella el último valor obtenido, variando sólo dos parámetros, y, por ello, se mueve por líneas, en cada una de las cuales queda libre un parámetro diferente, entrando así en iteración, en la proximidad del valor óptimo buscado. Por ser bastante próximo el valor entregado por el segundo código, el trabajo del tercer código no es sino un ajuste fino de la convergencia y sólo para algunos parámetros a ajustar. Por otra parte, en ocasiones, el uso del tercer código resulta superfluo, pues con los dos primeros se ha llegado a una solución exacta y suficientemente precisa.

\section{ALGORITMOS EMPLEADOS}

El algoritmo básico para el primer código de búsqueda de valores iniciales pasa por ajustar el modelo:

$$
Z_{\mathrm{i}}=a_{0}+a_{1} \cdot X_{1 . \mathrm{i}}+a_{2} \cdot X_{2 . \mathrm{i}}\left(a_{3 . \mathrm{k}}\right)+e_{\mathrm{i}}
$$

donde

$Z_{\mathrm{i}}=\ln [\dot{\epsilon}], a_{0}=\ln [A], a_{1}=-Q, X_{1 . \mathrm{i}}=1 / R \cdot T_{\mathrm{i}}, a_{2}=$ $n, X_{2 \cdot \mathrm{i}}=\ln \left[\operatorname{senh}\left[a_{3 \cdot \mathrm{k}} \cdot \sigma_{\mathrm{i}}\right]\right]$, con $a_{3 \cdot \mathrm{k}}=\alpha_{\mathrm{k}} ;$ siendo $R=$ constante de los gases perfectos. La función objetivo se ajusta mediante una regresión por mínimos cuadrados lineales al conjunto de datos $\left\{\dot{\epsilon}_{\mathrm{i}}, \sigma_{\mathrm{i}}, T_{\mathrm{i}}\right\}$, $i=1 \ldots n$, recorriendo $a_{3 \cdot \mathrm{k}}$ un intervalo $\left[a_{3 \cdot \mathrm{min}}\right.$, $\left.a_{3 \text { máx }}\right]$, con tamaño de paso $\Delta a_{3}$. Para cada valor $K=1 \ldots p$ de la constante $a_{3}$, se obtiene un vector de estimadores $\{\alpha\}_{i=0 \ldots 2}$, mediante $\alpha=$ $\left(X^{\prime} \cdot X\right)^{-1} \cdot X^{\prime} \cdot Z$, donde $X^{\prime}$ indica trasposición de matrices y la notación es la tradicional en estos $\operatorname{casos}\left(X=\left\{x_{\mathrm{i} \cdot \mathrm{j}}\right\}, i=0,1,2 j=1 \ldots n\right.$ matriz de datos, $Z=$ vector de datos). La construcción del algoritmo, los controles estadísticos y el análisis de los residuos, así como la tabla ANOVA y la aplicación de las validaciones de hipótesis pertinentes $(F$ Snedecor y $t$-Student) se pueden consultar en un artículo anterior (5). La determinación del óptimo se hace sobre el coeficiente de determinación $R^{2} \mathrm{k}$, que varía con $\{k\}$ al igual que $a_{3 \cdot \mathrm{k}}$, siendo $R^{2}{ }_{\mathrm{k}}=$ $\left(\Sigma\left(Z_{\mathrm{i}, \text { est }}-E(Z)\right)^{2}\right) /\left(\Sigma\left(Z_{\mathrm{i}}-E(Z)\right)^{2}\right)$, con $Z_{\mathrm{i} \text {,est }}=X . \alpha$. Seleccionado el valor máximo del coeficiente de determinación, después de analizar y depurar el conjunto de datos experimentales y comprobada la bondad del ajuste, se crea un fichero con los resultados para $\{A, Q, n, \alpha\}$ que será utilizado por el código siguiente como conjunto de valores iniciales. El algoritmo siguiente trata de forma no lineal el problema. Dado $Z(i)=\mathrm{f}\left(X(i)_{\mathrm{k}}, \alpha^{0}\right)+e(i)$, planteado en forma matricial para $i=1,2, \ldots, n$ (datos) y $\alpha^{0} \in \mathbb{R}^{4}$, aplicamos un minimizador

$$
\operatorname{SSE}(\alpha)=\|Z-f(\alpha)\|^{2}
$$

siendo el estimador que garantiza el óptimo en la aproximación del método de Gauss-Newton modificado:

$$
\hat{\alpha}=\alpha_{0}+\left(F^{\prime} \cdot F\right)^{-1} F^{\prime} \cdot e+O p \frac{1}{\sqrt{n}}
$$


siendo $f(\alpha)=\alpha_{0} \cdot e^{(-\alpha 1 / X(1, \mathrm{i}))} \cdot\left[\operatorname{senh}\left(\alpha_{3} \cdot X(2, i)\right)\right]^{\alpha 2}$, las variables $Z$ y $X$ son las indicadas anteriormente, $F$, indica trasposición y la matriz $F$ es el gradiente respecto de la variable $\alpha$. A partir de los valores iniciales aportados por el código anterior, se procede a un método de avance en la dirección del gradiente con una optimización lineal univariada para cada paso y con la aplicación de cuidadosos sistemas de control de la velocidad y de la convergencia. Además, el problema se trata con controles estadísticos adecuados, análisis de residuos y estudio de la bondad del ajuste. Los detalles se pueden consultar en un artículo anterior (6). En ocasiones, el trabajo conjunto de los dos anteriormente detallados es suficientemente preciso para detener el trabajo y tomar como óptimo el resultado obtenido. No obstante, en otras ocasiones no es así y pese a haber obtenido un valor, éste no es el óptimo, pues está condicionado por el desarrollo seguido; por esto se ha construido un método iterativo que trabaja a partir del último resultado, realizando un ajuste fino del resultado. Este código esta formado, fundamentalmente, por dos rutinas encadenadas de forma iterativa, en cada una de ellas se realiza una regresión lineal de la forma $Z A(i)=A P+n \cdot X A(i)$, siendo en cada una diferentes las variables. En la primera $Z A(i)=Z(i)+Q / X(2, i)$, con $Z(i)=\ln [\dot{\epsilon}(i)], \quad X(2, i)=R \cdot T(i)$, $A P=\operatorname{Ln}[A], n$ tiene el significado dado en la ec. [1], $X A(i)=\ln [\operatorname{sen} h[\alpha(k) \cdot X(1, i)]]$, con $X(1, i)=\sigma(i)$, todas las notaciones no especificadas tienen el significado dado en la ec. [1]. En esta rutina $\alpha(k)$ recorre todos los valores de $K$, resultantes de discretizar el intervalo de valores físicamente admisibles con un paso adecuado para que el resultado del valor óptimo garantice una precisión de 0,001 . Se determina el valor óptimo a partir del coeficiente de determinación y se graban los valores del óptimo en un fichero que toma la rutina siguiente, en la cual $Z A(i)=\ln [A]+Q(k) / X(2, i), X A(i)=\ln [\operatorname{sen} h[\alpha \cdot X(1, i)]]$, y todos los demás valores son los mismos. En esta rutina, la constante que se discretiza y sobre la que se realizan regresiones progresivas es el parámetro $Q(k)$, lo cual se hace con el mismo criterio de precisión indicado. En la primera $Q=$ cte. y en la segunda $\alpha=$ cte. Los detalles del control de iteraciones, variación de rangos de recorridos y ajuste de precisiones, se pueden ver en dos trabajos anteriores ( 5 y 6 ).

\section{APLICACIONES, MATERIAL Y PROCEDIMIENTO EXPERIMENTAL}

Para controlar el funcionamiento del conjunto de códigos, se han utilizado dos tipos de materiales. Un acero microaleado con carbono y un pequeño porcentaje de titanio, y el segundo material que es la bien conocida aleación Ti-6Al-4V, que es una de las aleaciones más ampliamente estudiadas por su interés como material estructural en aplicaciones aeroespaciales. El acero microaleado ha sido caracterizado en diversos trabajos: García et al. (7), Peñalba et al. (8 y 9). La aleación de titanio ha sido previamente estudiada por Peñalba et al. (10) y Carsí et al. (11). Los datos experimentales se han obtenido mediante validación de la torsión en un banco de torsión descrito en (8); los datos experimentales obtenidos se presentan en las tablas I y II, y las características del acero en la tabla III.

La muestra de titanio está formada por 33 datos, lo que es estadísticamente representativo para este tipo de trabajos. La de acero está formada por 16 datos, por lo que de presentarse algún tipo de problema en los mismos haría que la muestra fuese pobre o escasa. En el titanio se trabaja con bajas velocidades de deformación, mientras que en el caso del acero se trabaja con valores altos. Los recorridos para temperaturas y tensiones son similares.

\section{RESULTADOS}

Para el acero microaleado y el titanio, la aplicación de la secuencia de los tres procesos presenta el siguiente resultado (Tablas IV, V y VI).

Las validaciones de hipótesis para la bondad del ajuste en el caso del acero y para el código de ajuste no lineal presentan un valor de la función $F$ de Snedecor experimental y con los parámetros del caso de 751,72, lo cual hace significativo el ajuste. El estudio de los residuos permite afirmar que, aun-

TABLA I.- Datos experimentales del acero microaleado

TABLE I.- Experimental data for the microalloyed steel

\begin{tabular}{|c|c|c|}
\hline$\dot{\epsilon}, \mathrm{s}^{-1}$ & $T, \mathrm{~K}$ & $\sigma, \mathrm{MPa}$ \\
\hline 2 & 1.353 & 75,76 \\
2 & 1.398 & 64,75 \\
7 & 1.353 & 96,21 \\
7 & 1.398 & 80,18 \\
14 & 1.353 & 107,2 \\
14 & 1.398 & 91,87 \\
21 & 1.353 & 113,71 \\
21 & 1.398 & 99,59 \\
1 & 1.423 & 53,55 \\
14 & 1.423 & 86,07 \\
21 & 1.423 & 91,69 \\
7 & 1.100 & 260,74 \\
2 & 1.373 & 67,63 \\
7 & 1.373 & 84,83 \\
14 & 1.373 & 97,12 \\
21 & 1.373 & 107,2 \\
\hline
\end{tabular}


TABLA II.- Datos experimentales para el titanio

TABLE II.- Experimental data for titanium

\begin{tabular}{|l|c|c|}
\hline \multicolumn{1}{|c|}{$\dot{\epsilon}, \mathrm{s}^{-1}$} & $T, \mathrm{~K}$ & $\sigma, \mathrm{MPa}$ \\
\hline 0,00007 & 1.073 & 23,91 \\
0,0002 & 1.073 & 43,92 \\
0,0005 & 1.073 & 73,75 \\
0,001 & 1.073 & 104,12 \\
0,003 & 1.073 & 147,69 \\
0,0002 & 1.123 & 12,82 \\
0,0005 & 1.123 & 223,08 \\
0,001 & 1.123 & 35,76 \\
0,003 & 1.123 & 56,37 \\
0,007 & 1.123 & 80,23 \\
0,0002 & 1.173 & 8,38 \\
0,0005 & 1.173 & 15,33 \\
0,001 & 1.173 & 21,41 \\
0,003 & 1.173 & 32,48 \\
0,003 & 1.173 & 52,59 \\
0,0005 & 1.179 & 9,59 \\
0,001 & 1.179 & 15,78 \\
0,003 & 1.179 & 25,34 \\
0,007 & 1.179 & 35,44 \\
0,07 & 1.200 & 72,3 \\
0,2 & 1.200 & 91 \\
2 & 1.200 & 169 \\
0,07 & 1.220 & 63,9 \\
0,2 & 1.230 & 67,4 \\
0,5 & 1.230 & 95,4 \\
2 & 1.230 & 131,3 \\
1 & 1.230 & 112,4 \\
0,5 & 1.250 & 68,6 \\
2 & 1.250 & 104 \\
5 & 1.250 & 130 \\
0,07 & 1.243 & 39 \\
0,2 & 1.270 & 51,5 \\
0,5 & 1.270 & 67,6 \\
\hline
\end{tabular}

que en alguna zona se detecta un alejamiento de la normalidad, globalmente se comportan correctamente (los datos). No se detectan outliers. Parece que los residuos presentan una cierta correlación serial (consecuencia del método de trabajo experimental). La función de densidad (en frecuencias) de los residuos se puede ver en las figuras 1 y 2 . La representación seriada de los residuos se muestra en las figuras 3 y 4 . Los resultados de la representación gráfica normal de la validación Chi-cuadrado

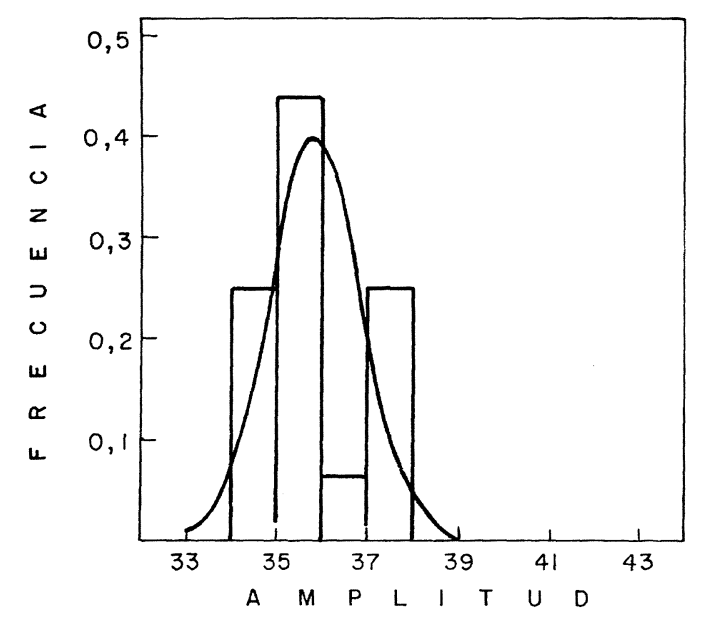

FIG. 1.- Una distribución de residuos para acero microaleado.

FIG. 1.- Residuals distribution for the microalloyed steel.

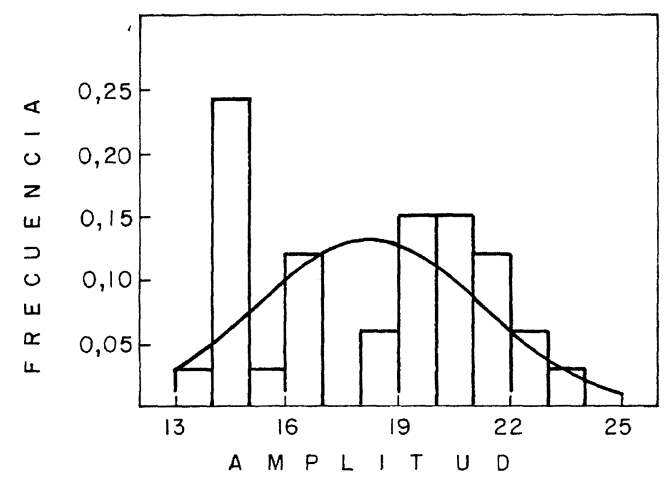

FIG. 2.- Una distribución de residuos para la aleación de titanio.

FIG. 2.-Distribution of residuals for the titanium alloy.

para los residuos se muestran en las figuras 5 y 6 . En la figura 7 se puede observar el comportamiento, si cabe errático o aleatorio, de los valores del coeficiente de determinación para los recorridos del tercer código por el método iterativo. Este comportamiento indica que los métodos iterativos con superficies de respuesta tan complejas como es la del trabajo presente pueden tomar líneas de avance que, muy próximas al mejor camino, lleven a soluciones no óptimas, como así sucede en muchas oca-

TABLA III.- Composición química del acero utilizado

TABLE III.-Chemical composition of the steel used

\begin{tabular}{|c|c|c|c|c|c|c|c|c|c|c|c|c|c|c|}
\hline $\mathrm{C}$ & $\mathrm{Mn}$ & $\mathrm{Si}$ & $\mathrm{S}$ & $\mathrm{P}$ & $\mathrm{Cr}$ & $\mathrm{Ni}$ & $\mathrm{Mo}$ & $\mathrm{V}$ & $\mathrm{Ti}$ & $\mathrm{Cu}$ & $\mathrm{Sn}$ & $\mathrm{Al}$ & $\begin{array}{c}\mathrm{N}, \\
\mathrm{ppm}\end{array}$ & $\begin{array}{c}\mathrm{O}, \\
\mathrm{ppm}\end{array}$ \\
\hline 0,29 & 1,28 & 0,34 & 0,03 & 0,02 & 0,13 & 0,08 & 0,01 & 0,09 & 0,02 & 0,13 & 0,02 & 0,04 & 106 & 45 \\
\hline
\end{tabular}


TABLA IV.- Resultados por código para el acero microaleado

TABLE IV.-Results by code for the microalloyed steel

\begin{tabular}{|c|c|c|c|c|c|}
\hline Material & $A$ & $Q, \mathrm{~J} / \mathrm{mol}$ & $N$ & $\alpha$ & $R$, (Coef, det.) \\
\hline Código 1 & $3,16 \cdot 10^{19}$ & $277.028 \pm 13.900$ & $5,48 \pm 0,24$ & $3,65 \cdot 10^{-4}$ & 0,9957 \\
Código 2 & $3,161 \cdot 10^{19}$ & 276.900 & 5,47 & $3,65 \cdot 10^{-4}$ & \\
Código 3 & $4,46 \cdot 10^{26}$ & 275.931 & 5,46 & $1,8 \cdot 10^{-5}$ & 0,9989 \\
\hline
\end{tabular}

TABLA V.- Resultados por código para la aleación de titanio

TABLE V.-Results by code for the titanium alloy

\begin{tabular}{|c|c|c|c|c|c|}
\hline Material & $A$ & $Q, \mathrm{~J} / \mathrm{mol}$ & $N$ & $\alpha$ & $R$, (Coef, det.) \\
\hline Código 1 & $7,08 \cdot 10^{16}$ & $422.871 \pm 22.200$ & $1,96 \pm 0,12$ & 0,016 & 0,993 \\
Código 2 & $7 \cdot 10^{16}$ & 422.867 & 194 & 0,016 & \\
Código 3 & $4,22 \cdot 10^{15}$ & 372.671 & 2,01 & 0,005 & $0,981(1)$ \\
& $5 \cdot 10^{16}$ & 419.000 & 1,96 & 0,016 & $0,982(2)$ \\
\hline
\end{tabular}

TABLA VI.- Resultados comparados

TABLE VI.-Compared results

\begin{tabular}{|l|c|c|c|c|c|}
\hline Material (Ref.) & $N$ & $Q, \mathrm{~J} / \mathrm{mol}$ & $\Delta T, \mathrm{~K}$ & $\Delta \dot{\epsilon}, \mathrm{s}^{-1}$ & Tipo de ensayo \\
\hline $\operatorname{Ti}\left({ }^{*}\right)$ & 1,97 & 419.000 & $1.073-1.270$ & $7 \cdot 10^{-5}-5$ & torsión \\
$\operatorname{Ti}(12)$ & 2,03 & 451.000 & $1.173-1.243$ & $7 \cdot 10^{-2}-8,7$ & torsión \\
$\operatorname{Ti}(13)$ & 2,0 & 400.000 & $1.073-1.173$ & $10^{-3}-10^{-1}$ & compresión \\
$\operatorname{Ti}(14)$ & 1,56 & 522.000 & $1.073-1.223$ & $5 \cdot 10^{-2}-50$ & torsión \\
Acero(*) & 5,46 & 275.931 & $1.100-1.423$ & $1-21$ & torsión \\
Acero $(15)$ & 4,76 & 339.000 & $1.323-1.473$ & $1-14$ & torsión \\
\hline
\end{tabular}

siones. Por el contrario, si se está próximo al valor óptimo y se itera por líneas adecuadas, el resultado será correcto. Sin embargo, los dos primeros, trabajando en conjunto, garantizan, además de más y mejor información, un resultado global y generalmente más correcto. En las figuras 8 y 9 se pueden observar las representaciones de la ecuación de Garofalo en tres dimensiones para las soluciones aportadas por nuestros códigos.

\section{CONCLUSIONES}

La comparación con los datos bibliográficos y con las condiciones físicas del problema permiten afirmar que los resultados obtenidos no sólo son buenos, sino que son más completos y se aportan con precisión, pudiendo determinar intervalos de confianza individuales o conjuntos para las cons- tantes obtenidas. El análisis estadístico de los códigos permite depurar las bases de datos a posteriori, eliminando de forma objetiva aquellos datos que podrían perturbar el resultado, pero no haciéndolo de forma arbitraria, con lo que se perdería información. En conjunto, el paquete de programas trabaja sin necesidad de valores iniciales, por lo que aporta una autoconsistencia grande a la resolución del problema. Aunque las características de los conjuntos de datos presentan problemas especiales, se alcanza una precisión suficiente, al menos en el marco de las necesidades actuales de la investigación aplicada. La exactitud de las soluciones es cuestionable en cualquier caso y está relacionada con el mayor o menor grado de verosimilitud de la ecuación de Garofalo. Cualquier profundización teórica en la actualidad, sobre los modelos que soportan dicha ecuación, pone de manifiesto que es preciso elegir ecuaciones más completas, más com- 


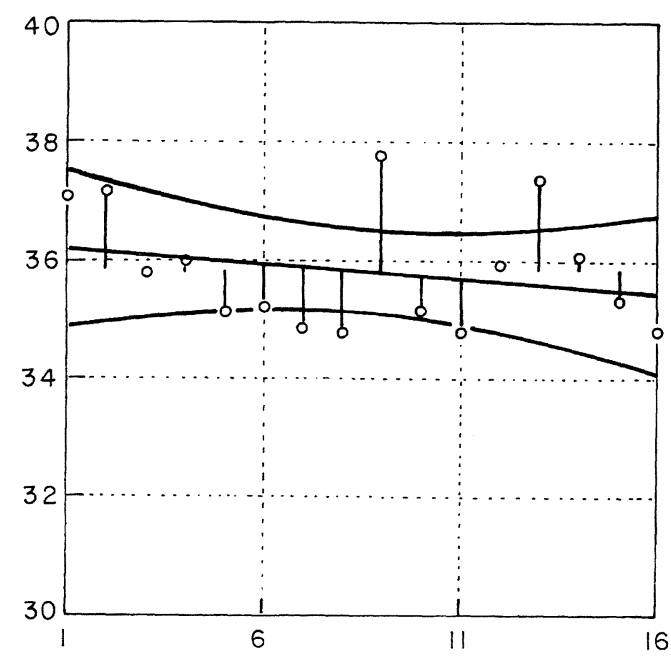

FIG. 3.- Residuos del ajuste para el acero microaleado.

FIG. 3.- Residuals of the fitting for the microalloyed steel.

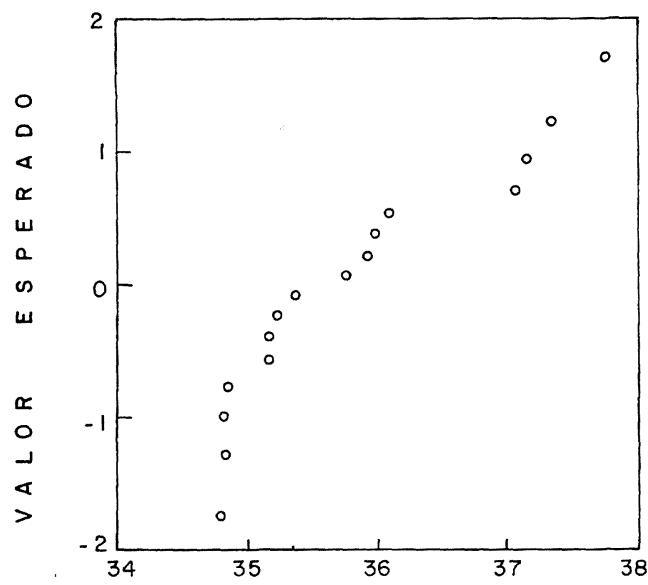

FIG. 5. - Ensayo de normalidad para el ajuste sobre residuos del acero microaleado.

FIG. 5.- Normality test for the fitting on residuals for the microalloyed steel.

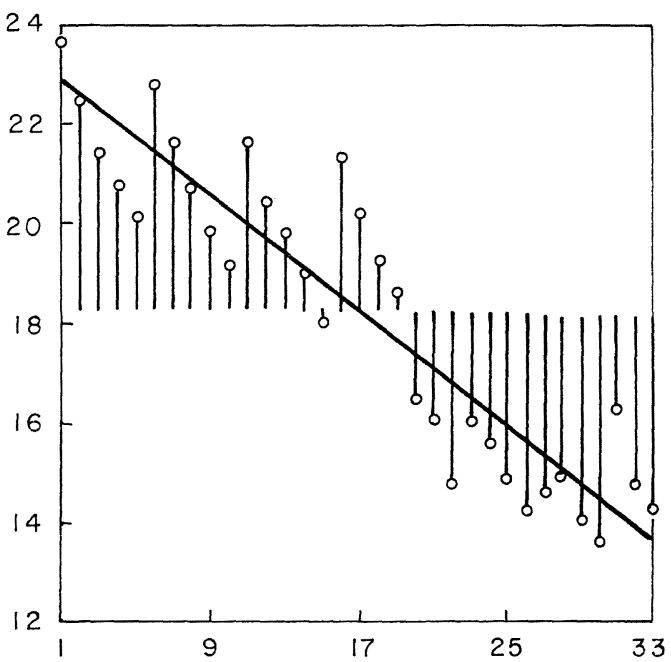

FIG. 4.- Residuos del mejor ajuste para la aleación de titanio.

FIG. 4.- Residuals of the best fitting for the titanium alloy.

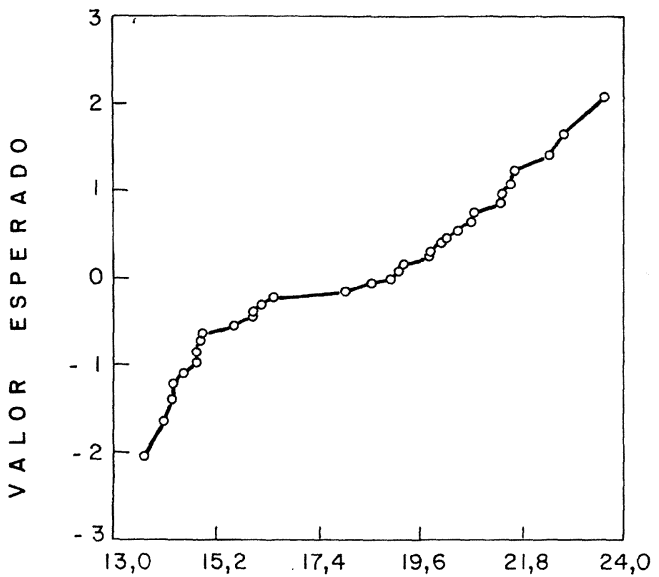

FIG. 6. - Ensayo de normalidad para el ajuste sobre residuos de la aleación de titanio.

FIG. 6.- Normality test for the residuals fitting of the titanium alloy.

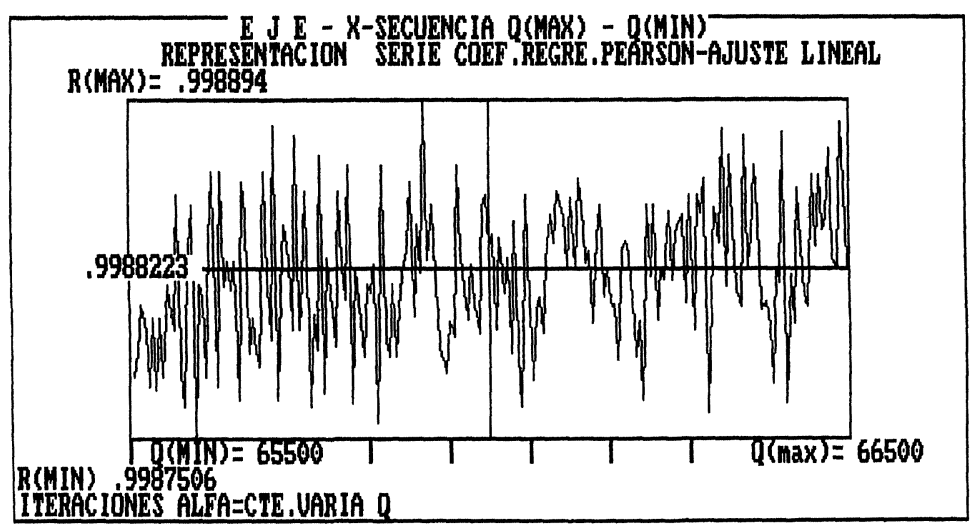

FIG. 7.- Variación del coeficiente de correlación en la zona solución.

FIG. 7.-Behaviour of the correlation coefficient around the solution. 


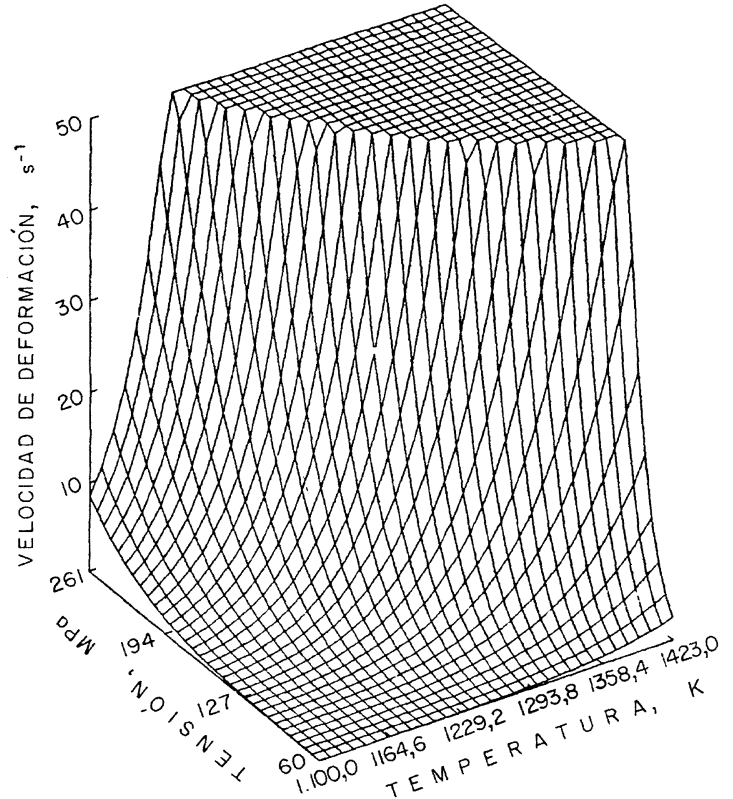

FIG. 8.- Representación tridimensional de la ecuación de Garofalo para el mejor ajuste de los datos del acero microaleado.

FIG. 8.- 3D representation of the Garofalo's equation for the best fitting of the experimental data (microalloyed steel).

plejas y quizás con modificaciones en su estructura. Diversos modelos presentan relaciones seno hiperbólicas diferentes y se haría preciso tener en cuenta que en algunos rangos de trabajo pueden coexistir diferentes fenómenos que contribuyen a la fluencia. Pese a todo lo anterior, parece que los métodos directos no lineales son una garantía de estar trabajando con mayor seguridad que con los métodos iterativos.

El CENIM puede proporcionar la solución de la ecuación de Garofalo a quienes lo soliciten sin que sea necesario requisito alguno más que enviar el conjunto de datos experimentales que se desea correlacionar.

\section{Agradecimiento}

Este trabajo ha sido realizado con la ayuda económica proporcionada por los programas Mat951736-E y Mat95-186..

\section{REFERENCIAS}

(1) Powell, M.J.D. Dpt. of Applied Mathematics on Theoretical Physics. Univ. Cambridge. Silver Street. Cambridge CB39EW (R.U.), págs. 122-141. Proc. Numerical Analysis. 10th Biennal Conf. Dundee (Escocia), 28 jun.-1 jul. 1993.

(2) FletCher, R. y ReEves, C.M. Comput. J., 7, 1980: 149-154.

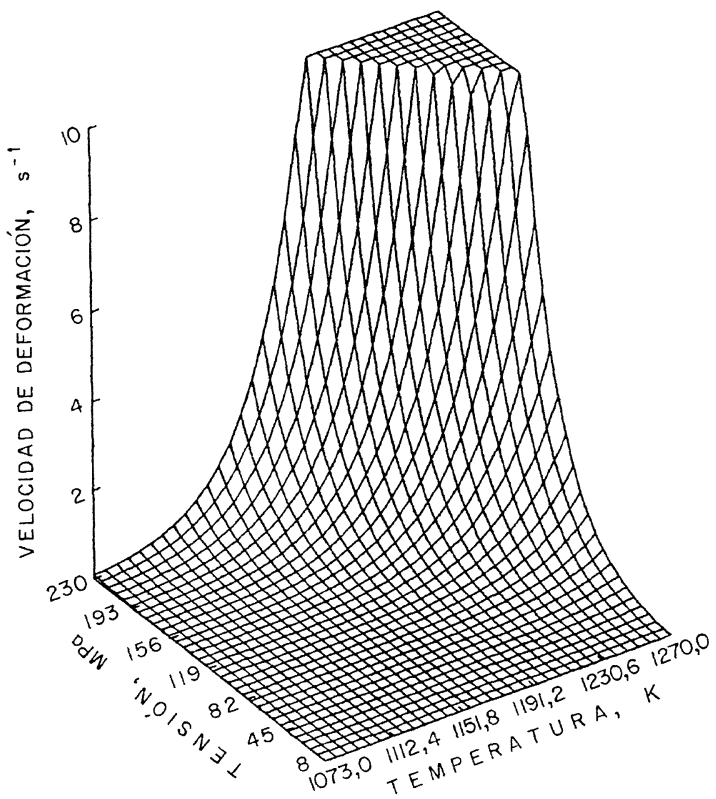

FIG. 9.- Representación tridimensional de la ecuación de Garofalo para el mejor ajuste de los datos de la aleación de titanio.

FIG. 9.- $3 D$ representation of the Garofalo's equation for the best fitting of the experimental data (titanium alloy).

(3) Botes D.M. y WatTs D.G. Nonlinear Regression Analisys and its Applications. Ed. John Wiley \& Sons. Nueva York (EE.UU.) 1993.

(4) Gallant, A.D. Nonlinear Statistical Models. Ed. John Wiley \& Sons. Nueva York (EE.UU.) 1986.

(5) Rieiro, I. y CARsí, M. Trabajo remitido a la revista A.L.I.O.

(6) Rieiro, I. y Carsí, M y Peñalba, F. Computational Methods and Testing for Engineering Integrity. Ed. T.V. Duggan. Southampton (R.U.), 1996: 301.

(7) García, C., Carsí, M., Peñalba, F. y de Andrés, M.P. J. Mater. Sci., 27 (17), 1992: 4.567-4.576.

(8) Peñalba, F., Carsí, M. y Zapirain, F. Mem. Sci. Rev. Metall. 99 (2), 1991: 79-90.

(9) Peñalba, F., Carsí, M., García, C., de Andrés, M.P. y ZAPIRAIN, F. ISIJ Intern., 32 (2), 1992: 232- 240.

(10) Peñalba, F. y Carsí, M. J. Mater. Sci., 30, 1995: 1.7501.753.

(11) Carsí, M., Peñalba, F. e Ibañez, J. G.G.D. Mater. Lett., 20, 1994: 119-123.

(12) VARGA, D. Central Research Institute for Physics. Hungarian Academy of Science. Budapest (Hungría): 161-166. Colloquia Mathematics of Societatys Janos Bolyai. Colloquiem on Numerical Methods in Titany (Hungría) 2-6 sept. 1968. 